\title{
General Two-Fluid Interacting \& Non-interacting Cosmological Models Theory
}

\section{Sanjay $0 \mathrm{Oi}^{1 *}$ and Dheemanth URS $\mathrm{R}^{2}$}

${ }^{1}$ Department of Mathematics, Dayananda Sagar College of Engineering, Bangalore, India ${ }^{2}$ Department of Information Science, Dayananda Sagar College of Engineering, Bangalore, India

*Corresponding author: Sanjay Oli, Department of Mathematics, Dayananda Sagar College of Engineering, Shavige Malleswara Hills, Kumaraswamy Layout, Bangalore- 560078, India, Email: olisanjay@gmail.com

\section{Abstract}

In this paper we begin with the standard cosmological model of universe \& present picture of current universe by observational results. A general theory of two-fluid cosmological models has been presented where one fluid portray the dark energy content and other fluid represent the dark matter content of universe. A detail overview of two-fluid cosmological models has been studied with proposed future space based observations.

Keywords: Einstein's field equations; Two-fluid; Interacting \& non-interacting; Dark energy; Dark matter

\section{Introduction}

In the standard cosmological model, the universe came into existence from a space-time singularity of infinite density as predicted by Einstein's general theory of relativity. The expansion started from a state of infinite density, which we call the big bang singularity. The laws of physics break down at the singularity. The big bang models are based on the theory of relativity and stand in good agreement of present observation on large angular scales. These models are based on assumptions that space-time is homogeneous and isotropic. The assumptions of homogeneity imply that all points of space are equivalent and properties associated with each point are the same, whereas the isotropy means that there is no privileged direction in space.

Cosmology has been a hub of theoretical research activities since the beginning of the twentieth century. A majority of these investigations has been much of a speculative nature due to the very reason that the subject had a meager observational backing till the early nineties. The modern study of cosmology was initiated by Einstein [1] and De Sitter [2] in 1917 who proposed a static model of the universe. The concept of an expanding universe was introduced by Fridemann [3] and Lamaitre [4]. Expanding models of the universe gained considerable interest after the discovery velocity-distance linear relationship among galaxies due to E. Hubble [5] in 1929, given by

$$
\mathrm{V}=\mathrm{c} \mathrm{Z}=\mathrm{H}_{0} \mathrm{D}
$$

where $\mathrm{D}$ is the distance of the galaxy and $\mathrm{Z}$ is its redshift and $\mathrm{H}_{0}$ is known as the Hubble constant. The value of $\mathrm{H}_{0}$ is believed to be $100 \mathrm{~h}_{0} \mathrm{kms}^{-1} \mathrm{Mpc}^{-1}$, where $\mathrm{h}_{0}$ lies between 0.5 to 1. Hubble's law implies that galaxies are receding each other and the recession is directly proportional to distance.

Another landmark discovery in the field of cosmological observation is the detection of cosmic microwave background radiation (CMBR) by Penzias and Wilson in 1965 [6]. These are two notable observational pillars on which stood the entire speculative edifice of theoretical cosmology and supports the Big Bang model.

Thehistory of cosmologyhas witnessed many conjectures. The latest is accelerated expansion of the universe indicating that in present universe the dominant energy components are dark energy and dark matter. Dark energy and dark 
matter are the cornerstones of modern cosmology and it has not yet been directly or indirectly detected but searches are currently under way. The presence of dark energy revealed by the recent speeding up of the expansion of the universe. In observational point of view, this discovery confirmed by the fluctuations of Cosmic Microwave Background Radiation (CMBR) $[7,8]$ observation of Large Scale Structure(LSS) [9], nine years cosmological results by WMAP $[10,11]$ and PLANK(XVI) [12] measurements that dark energy and dark matter are basic constituents of the universe.

The observational results by Baryon Oscillation Spectroscopy Survey(BOSS) [13]; Abell cluster A586 [14]; optical, X ray and weak lancing data from 33 relaxed galaxy clusters [15] find out the signature of coupling between dark matter and dark energy. Hence, one could investigate cosmological models in which dark energy and dark matter interact each other. Also, interacting dark energy and dark matter models are known to solve the coincidence problem (why the densities of dark matter and dark energy are same order at present epoch?). Recently, the results by Shah \& Iqbal confirms that gravitational collapse of dark energy and dark matter with interaction leads to the formation of the black hole $[16,17]$.

The projects HETDEX - the Hobby-Eberly Telescope Dark Energy Experiment, DES: Dark Energy Survey, LSST: Large Synoptic Survey Telescope, WFIRST: Wide-Field Infrared Survey Telescope \& Euclid has been planned for the next generation search to provide a more complete picture of the history of the universe and the role of dark energy and dark matter.

\section{General Theory of Two-fluid Models}

The infinitesimal distance between two adjacent points in a four dimensional Riemannian space-time is given by

$$
d s^{2}=g_{i j} d s^{i} d s^{j}
$$

where the latin suffixes $i$ and $j$ take values in the range 1,2,3 and 4. Here $g_{i j}$ is the metric tensor; $\mathrm{x}^{1}, \mathrm{x}^{2}, \mathrm{x}^{3}$ denote spacelike coordinate and $x^{4}$ the time like coordinate. A semicolon followed by a suffix denotes covariant differentiation with respect to $g_{i j}$.

Einstein's general relativistic field equations for non empty space-time are given by

$$
G_{i j}=-\frac{8 \pi G}{c^{4}} T_{i j}
$$

where

$$
G_{i j} \equiv R_{i j}-\frac{1}{2} g_{i j} R+\Lambda g_{i j}
$$

is the Einstein tensor. $R_{i j}$ is the Ricci tensor, which is symmetric in i and $j$ and expresses the curvature of the spacetime region. It is given by

$$
R_{i j}=\frac{\partial}{\partial x^{j}} \Gamma_{i k}^{k}-\frac{\partial}{\partial x^{k}} \Gamma_{i j}^{k}+\Gamma_{i k}^{a} \Gamma_{j i}^{k}-\Gamma_{i j}^{a} \Gamma_{a k}^{k}
$$

where

$$
\Gamma_{i j}^{k}=\frac{g^{a k}}{2}\left(\frac{\partial}{\partial x^{i}} g_{j a}+\frac{\partial}{\partial x^{j}} g_{i a}-\frac{\partial}{\partial x^{a}} g_{i j}\right)
$$

are Christoffel symbols of second kind and

$$
g^{i j} g_{i j}=\delta_{k}^{j}=\operatorname{diag}(1,1,1,1)
$$

$R \equiv g^{i j} R_{i j}$ is the scalar curvature and $\mathrm{G}$ is the Newtonian constant of gravitation. In C.G.S. units $\mathrm{G}=6.67 \times 10^{-8} \mathrm{~cm}^{3} \mathrm{~g}^{-1} \mathrm{~s}^{-2}$ and $\mathrm{c}=3 \times 10^{10} \mathrm{~cm} \mathrm{~s}^{-2} . \Lambda$ is the cosmological constant. It was

introduced by Einstein in the field equations in order to get a static model of the universe. The interpretation is that the positive value of cosmological constant introduces a repulsive force at every point of the space, which can counterbalance the attractive force of gravity and vice-versa.

$T_{i j}$ is the energy-momentum tensor, represents the total non-gravitational energy content in a unit proper three volume. For a perfect fluid of isotropic pressure $p$ and density

$\rho, T_{i j}$ is given by

$$
T_{i j}=\left(p+\rho c^{2}\right) u_{i} u_{j}-p g_{i j}
$$

where the four-velocity, $u_{1}$ satisfies the equation (7).The conservation of energy-momentum tensor for Einstein field equations implies that,

$$
T_{j, i}^{i}=0
$$

In 1917, static, homogeneous and isotropic cosmological models were proposed by Einstein [1] and DeSitter [2]. In Einstein space-time hyper surfaces $t=$ constant are filled with incoherent matter of constant density. It satisfies Mach principle and the density of matter determines the precise geometrical nature of the space-time. The DeSitter universe is an empty space-time that is in the dynamical sense the universe is empty, whereas in the kinematical sense it is expanding. Eddington pointed out that; DeSitter universe has motion without matter in respect to Einstein universe which has matter without motion. Both the models have their own advantages. So, various author's recognized the need of cosmological models of universe, which contain the 


\section{Physical Science \& Biophysics Journal}

advantages of both of these models.

\section{Cosmological Models which contain both Matter and Radiation}

The discovery of isotropic cosmic microwave background radiation encouraged many cosmologists to investigate cosmological models which contain both matter and radiation. In a two-fluid model, each of the density and the isotropic pressure has two components:

$$
\rho=\rho_{r}+\rho_{m}, p=p_{r}+p_{m}
$$

where subscripts $m$ and $r$ respectively correspond to matter and radiation. The isotropic pressure of radiation is given by $p_{r}=1 / 3 \rho_{r} c^{2}$. Consequently, the energy momentum

tensor for a source of gravitational field consisting of a perfect fluid and diffused radiation, is given by

$$
T_{i j} \equiv T_{i j}^{(m)}+T_{i j}^{(r)}
$$

where

$$
\begin{gathered}
T_{i j}^{(m)}=\left(p_{m}+\rho_{m} c^{2}\right) u_{i}^{m} u_{j}^{m}-p_{m} g_{i j} \\
\& \\
T_{i j}^{(r)}=\frac{4}{3} \rho_{r} c^{2} u_{i}^{r} u_{j}^{r}-\frac{1}{3} \rho_{r} c^{2} g_{i j}
\end{gathered}
$$

Four- velocity $u_{i}^{m}$ and $u_{i}^{r}$ satisfy

$$
g^{i j} u_{i}^{m} u_{j}^{m}=g^{i j} u_{i}^{r} u_{j}^{r}=1
$$

The energy conditions are

$$
p_{m}+\rho_{m} c^{2}>0, \rho_{m}, \rho_{r}>0 .
$$

It has been observed that the cosmic microwave radiation has a black body spectrum. Therefore, its temperature $T_{r}$ is related to its density as follows:

$$
\rho=a T_{r}^{4}
$$

where $\mathrm{a}=7.57 \times 10^{-15} \mathrm{erg} \mathrm{cm}^{-3} \mathrm{~K}^{-1}$ is Stefan's constant. In two-fluid model, the law of conservation of energy and momentum leads to the equation

$$
T_{j, i}^{i^{(m)}}+T_{j, i}^{i(r)}=0,
$$

where

$$
T_{j, i}^{i}=\frac{\partial}{\partial x^{i}} T_{j}^{i}-T_{k}^{i} \Gamma_{i j}^{k}+T_{j}^{k} \Gamma_{i k}^{i} w i t h T_{i}^{j}=g^{i k} T_{k i}
$$

It is to note that the two fluids are interacting if they satisfy the unified conservation law given above. This situation is most relevant to portray the scenario before the recombination epoch when the photons were bound to matter. The two-fluids are non interacting if the corresponding energy-momenta are conserved separately:

$$
T_{j, i}^{i^{(m)}}=T_{j, i}^{i^{(r)}}=0 .
$$

The situation is generally considered to model the post recombination eras when the photons got themselves free to form CMBR being observed presently.

A general feature of the two-fluid model is that it expands out an absolute radiation phase towards a final matter phase. It is found that the inclusion of radiation in a matter universe will tend to decrease the age of universe, whereas inclusion of matter in a radiation universe will tend to decrease comic microwave radiation temperature [18].

\section{Cosmological Models which Contain Dark Matter and Dark Energy}

For two-fluid source, consisting a dark energy field and dark fluid source $T_{j}^{i}$ is,

$$
T_{j}^{i}=T_{j}^{i(d m)}+T_{j}^{i(d e)}
$$

where $T_{j}^{i(d m)}$ are $T_{j}^{i(d e)}$ the energy momentum-tensor for dark matter and dark energy field source.

For two-fluid model, the law of conservation of energy and momentum leads us,

$$
T ;{ }_{j}^{i j}=0
$$

From equation (20), it implies that

$$
T_{; j}^{i j(d m)}+T_{; j}^{i j(d e)}=0
$$

The two-fluids are interacting if they satisfy the unified conservation law (22) and non-interacting each other if the corresponding energy-momentum for dark matter and dark energy field source are conserved separately, that is

$$
T_{; j}^{i j(d m)}=T_{; j}^{i j(d e)}=0
$$

In the literature, it is usual to assume that dark energy and dark matter interact through a coupling term $Q$, given by

$$
\dot{\rho}_{d m}+3 H \rho_{d m}=Q \dot{\rho}_{d e}+3 H \rho_{d e}\left(1+w_{d e}\right)=-Q
$$


where $\rho_{d m}$ and $\rho_{d e}$ are densities of dark matter and dark energy respectively (ignoring the baryon component) and an overdot denotes differentiation with respect to comoving time t. $w_{d e}$ is the EoS parameter of dark energy given by $w_{d e}=p_{d e} / \rho_{d e}$ and $\mathrm{H}$ is the Hubble parameter.

Putting $Q=0$ in equation (24), the two-fluids becomes non-interacting each other and energy-momentum for dark matter and dark energy field source are conserved separately. It is be noted that, equation (24) preserve the total energy conservation equation given by (21) as follows,

$$
\dot{\rho}_{\text {Total }}+3 H \rho_{\text {Total }}\left(1+w_{\text {Total }}\right)=0
$$

where $\rho_{\text {Total }}=\rho_{d e}+\rho_{d m}$ is the total energy and $w_{\text {eff. }}$ is the total effective EoS.

For $Q>0$ or $Q<0$ expresses energy transfer from dark energy to dark fluid or dark fluid to dark energy. The forms of $Q$ considered in literature are completely arbitrarily, with

the obvious requirement to depend only on time due to homogeneity and isotropy. The usual choices of $Q$ are (i) $Q=\alpha \kappa \rho_{d m} \dot{\phi}$ (in string theory or scalar-tensor theory or Brans-dicke theory) $[19,20]$,

(ii) $Q=3 b^{2} H \rho_{M C G}$ (in Modified Chaplygin Gas)

(iii) $Q=3 \beta H\left(\rho_{d m}+\rho_{d e}\right)$ or $Q=3 \eta H \rho_{d m}$

Also, one can use observations in order to restrict their forms for dark energy EoS and effective EoS for dark energy and dark fluid [24].

\section{Conclusion}

Recent cosmological investigation by high red-shift IaSuper-nova (SN Ia) [25-27], Cosmic Microwave Background (CMB) Anisotropy [28], Large Scale Structure (LSS) [29] indicate that the expansion of the universe is expanding with acceleration and dark energy dominates in the present universe (4\% for baryonic matter,23\% for non baryonic dark matter and $73 \%$ of dark energy) [30]. Dark matter and dark energy are mysterious and we presently do not know what it is. By definition, dark matter has attractive gravity whereas dark energy is some kind of repulsive gravity with negative pressure.

This acceleration is realized with negative pressure and positive energy density that violates the strong energy condition. This violation of strong energy condition provides a reverse gravitational effect and universe gets jerk from its earlier deceleration phase to recent acceleration phase [9].

Recently, many authors studied two-fluid scenario in different geometries filled with barotropic fluid and dark energy [31-41]. We can get more information insight about nature of dark matter, dark energy and the interaction witin the dark sectors by the satellite mission Euclid and WFIRST scheduled for launch in 2020 and 2023.

Acknowledgements: I would like to thank the anonymous referee for fruitful suggestions.

\section{References}

1. Einstein A (1917) Kosmologische Betrachtungen zur allgemeinen Relativitätstheorie. Königlich Preußische Akademie der Wissenschaften (Berlin) Sitzungsberichte, 152.

2. De Sitter W (1917) On the Relativity of Inertia-Remarks Concerning Einstein's Latest Hypothesis. KNAW Proceedings, Amsterdam 19: 1217-1225.

3. Friedman A (1924) Über die Möglichkeit einer Welt mit konstanter negativer Krümmung des Raumes. Zeitschrift für Physik 21: 326-332.

4. Lemaitre AG (1931) A Homogeneous Universe of constant mass and increasing radius accounting for the radial velocity of extra-galactic Nebulae. Mon Not R Astr Soc 91(5): 483-490.

5. Hubble E (1929) A relation between distance and radial velocity among extra-galactic nebulae PNAS 15(3): 168173.

6. Penzias AA, Wilson RW (1965) A measurement of excess antenna temperature at $4080 \mathrm{MHz}$ Astrophysical J 142: 419-421.

7. Huang ZY, Wang B, Abdalla E, Ru-Keng S (2006) Holographic explanation of wide-angle power correlation suppression in the cosmic microwave background radiation. JCAP 5: 13.

8. Daniel SF, Robert RC, Asantha C, Melchiorri A (2008) Large scale structure as a probe of gravitational slip. Phys Rev D 77(10). 
9. Caldwell RR, Komp W, Parker L, Vanzella DAT (2006) Sudden gravitational transition Phys Rev 73: 023513.

10. Hinshaw G, Larson D, Komatsu E, Spergel DN, Bennett CL, et al. (2013) Nine-year Wilkinson Microwave Anisotropy Probe (WMAP) Observations: Cosmological parameter results. Astrophys J Suppl Series 208(2): 19.

11. Bennett CL, Larson D, Weiland JL, Jarosik N, Hinshaw G, et al. (2013) Nine-year Wilkinson Microwave Anisotropy Probe (WMAP) Observations: Final maps and results. Astrophys J Suppl Series 208(2): 20.

12. Ade PAR, Aghanim N, Armitage-Caplan C, Arnaud M, Ashdown M, et al. (2014) Planck 2013 results. XVI. Cosmological parameters. Astron \& Astrophy 571: 66.

13. Alam S, Ata M, Bailey S, Beutler F, Bizyaev D, et al. (2017) The clustering of galaxies in the completed SDSS-III Baryon Oscillation Spectroscopic Survey: cosmological analysis of the DR12 galaxy sample. Mon Not R Astr Soc 470(3): 2617-2652.

14. Bertolami O, Pedro FG, Delliou ML (2008) Dark EnergyDark Matter Interaction from the Abell Cluster A586. EAS Publication Series 30: 161-167.

15. Abdalla E, Abramo LR, Sodre LJ, Wang B (2009) Signature of the interaction between dark energy and dark matter in galaxy clusters. Phys Lett B 673(2): 107-110.

16. Shah HH, Iqbal Q (2017) Gravitational collapse of dark matter interacting with dark energy: Black hole formation. Int J Mod Phy D 26(13).

17. Shah HH (2018) Black hole formation due to collapsing dark matter in a presence of dark energy in the braneworld scenario. Int J Mod Phy D 27(3).

18. Coley AA, Tupper BOJ (1986) Two fluid FRW cosmologies and their numerical predictions. Can. J Phys 64(2): 204209.

19. Amendola L, Quercellini C (2003) Tracking and coupled dark energy as seen by the Wilkinson Microwave Anisotropy Probe. Phys Rev D 68(2).

20. Wei H, Tang NN, Zhang SN (2007) Reconstruction of hessence dark energy and the latest type Ia supernovae gold dataset. Phys Rev D75(4): 1-14.

21. Wang C, WU Ya-Bo, LIU (2009) Fei Evolution of Holographic Dark Energy in Interacting Modified Chaplygin Gas Model. Chin Phys Lett 26: 02980.

22. Guo ZK, Cai RG, Zhang YZ (2005) Cosmological evolution of interacting phantom energy with dark matter. JCAP, pp: 02.

23. Cai RG, Wang A (2005) Cosmology with interaction between phantom dark energy and dark matter and the coincidence problem JCAP, pp: 1-15.

24. Costa AA, Xu XD, Wang B, Ferreira EGM, Abdalla E (2014) Testing the interaction between dark energy and dark matter with Planck data. Phys Rev D 89: 103531.

25. Perlmutter S, Aldering G, Goldhaber G, Knop RA, Nugent $P$, et al. (1999) Measurements of Omega and Lambda from 42 High-Redshift Supernovae. Ap J 517: 565-586.

26. Perlmutter S, Aldering G, Della Valle M, Deustua S, Ellis RS, et al. (1998) Discovery of a supernova explosion at half the age of the universe. Nature 391: 51-54.

27. Coley A, Goldhaber G, Wang L, Aldering G, Amanullahet $R$, et al. (2006) Measurement of $\Omega_{\mathrm{M}}, \Omega_{\Lambda}$ from a blind analysis of Type Ia supernovae with CMAGIC: Using color information to verify the acceleration of the universe. Ap J Lett: 645: 1-20.

28. Hanany S, Ade P, Balbi A, Bock J, Borrill J, et al. (2000) MAXIMA-1: A Measurement of the Cosmic Microwave Background Anisotropy on Angular Scales $10^{\prime} 5^{\circ}$. Ap J 345(1).

29. Spergel DN, Verde L, Peiris HV, Komatsu E, Nolta MR, et al. (2003) First-Year Wilkinson Microwave Anisotropy Probe (WMAP) Observations: Determination of Cosmological Parameters. Astrophys J Suppl 148: 175194.

30. Baltay C (2014) The accelerating universe and dark energy. Int J Mod Phy D 23(6): 1-34.

31. Amirhashchi H, Pradhan A, Saha B (2011) An interacting two-fluid scenario for dark energy in a FRW universe. Chin Phys Lett 28(3): 1-4.

32. Pradhan A, Amirhashchi H, Saha B (2011) An interacting and non-interacting two-fluid scenario for dark energy models in a FRW universe with constant deceleration parameter. Astro Phys Sci 333: 343-350.

33. Saha B, Amirhashchi H, Pradhan A (2012) Two-fluid scenario for dark energy models in a FRW universe revisited. Astro Phys Sci 342: 257-267.

34. Amirhashchi H, Pradhan A, Zainuddin H (2013) Interacting two-fluid viscous dark energy models in a non-flat universe. Research in Astron Astrophys 13(2): 129-138.

35. Samanta GC (2013) Two-fluid anisotropic cosmological 
Physical Science \& Biophysics Journal

model with variable G and Lambda. Int J Theor Phys 52: 4015-4024.

36. Chirde VR, Shekh SH (2014) Interacting two-fluid viscous dark energy models in self creation cosmology. The African Rev of Phys 9: 399-409.

37. Verma MK, Chandel S, Shri Ram (2015) Anisotropic symmetric two-fluid cosmological model with selfcreation cosmology time-varying $\mathrm{G}$ and Lambda. Chinese Phy Lett 32: 120401.

38. Santhi VM, Rao VUM, Aditya Y (2016) Kaluza-Klein cosmological models with two fluids source in Brance-
Dicke theory of gravitation. African Rev Phys 11: 0029.

39. Garg P, Zia R, Pradhan A (201) Transit cosmological models in FRW universe under the two-fluid scenario. Int J Geome Meth Mod Phys 16(1): 195007.

40. Satish J, Venkateswarlu R (2019) Anisotropic Bianchi Type-VI two-fluid cosmological model coupled with massless sclar field and time-varying $G$ and Lambda. Bulg J Phys 46: 67-79.

41. Dagwal VJ, Pawar DD (2020) Two-fluid sources in F (T) theory of gravity. Mod Phy Lett A 35(4): 1950357. 